\title{
Non-normal parameter blowout bifurcation: An example in a truncated mean-field dynamo model
}

\author{
Eurico Covas, ${ }^{1, *}$ Peter Ashwin, ${ }^{2, \dagger}$ and Reza Tavakol ${ }^{1, \$}$ \\ ${ }^{1}$ Astronomy Unit, School of Mathematical Sciences, Queen Mary \& Westfield College, Mile End Road, London E1 4NS, United Kingdom \\ ${ }^{2}$ Department of Mathematical and Computing Sciences, University of Surrey, Guildford GU2 5XH, United Kingdom
}

(Received 11 June 1997)

\begin{abstract}
We examine global dynamics and bifurcations occurring in a truncated model of a stellar mean-field dynamo. This model has symmetry-forced invariant subspaces for the dynamics and we find examples of transient type I intermittency and blowout bifurcations to transient on-off intermittency, involving laminar phases in the invariant submanifold. In particular, our model provides examples of blowout bifurcations that occur on varying a non-normal parameter; that is, the parameter varies the dynamics within the invariant subspace at the same time as the dynamics normal to it. As a consequence of this we find that the Lyapunov exponents do not vary smoothly and the blowout bifurcation occurs over a range of parameter values rather than a point in the parameter space. [S1063-651X(97)00312-7]

PACS number(s): 05.45.+b
\end{abstract}

\section{INTRODUCTION}

There has recently been a great deal of work on deterministic dynamical systems possessing invariant submanifolds, motivated by symmetric systems, and in particular some coupled systems. Such systems have been shown to be capable of producing a range of interesting and robust dynamical modes of behavior, such as riddled basins [1] and on-off intermittency [2], shown to be related by the blowout bifurcation [3], as well as transient on-off intermittency [4]. We denote the manifold on which such a system is defined by $M$ and the corresponding invariant submanifold by $M_{I}$. An attractor is said to have a riddled basin if every open set intersecting the basin also intersects the basin of another attractor in a set of positive measure; such basins can arise robustly for Milnor attractors [5] contained in $M_{I}$.

On-off intermittency to a state in $M_{I}$ is characterized by dynamics that comes arbitrarily close to the state in $M_{I}$ but that also has intermittent large deviations from $M_{I}$. Transient on-off intermittency is a transient dynamics exhibiting characteristics of on-off intermittent behavior. Namely, it has an average distribution of laminar phases that satisfies a power law with exponent -3/2 [4]. All these phenomena arise as different aspects of blowout bifurcations, where a maximum normal Lyapunov exponent of an attractor in $M_{I}$ passes through zero and thus causes a loss of stability. To date, most mathematical understanding of such systems is limited to cases where there are a number of simplifying assumptions:

(H1) The control parameters are assumed to be normal [6] in the sense that the dynamics of the invariant submanifold is independent of the bifurcation parameter. Such parameters preserve the dynamics on the invariant submanifold, but allow it to vary in the rest of the phase space.

\footnotetext{
*Electronic address: E.O.Covas@qmw.ac.uk

${ }^{\dagger}$ Electronic address: P.Ashwin@mcs.surrey.ac.uk

‡Electronic address: reza@maths.qmw.ac.uk
}

(H2) The attractor that becomes transversely unstable in the $M_{I}$ and hence causes the blowout bifurcation is usually assumed to be chaotic.

(Note, however, that Yalcinkaya and Lai [7] find blowout type bifurcations from quasiperiodic dynamics on $T^{2}$.)

As many physical systems of interest are unlikely to possess normal parameters (a notable exception to this being some coupled systems), we expect that (H1) is not usually applicable. This is particularly expected to be true in truncations of partial differential equations that arise in fluid and dynamo equations (as well as astrophysical models in general). Thus, by studying the behavior of a specific example where (H1) does not hold, we hope to throw some light on the dynamics of general systems of this type.

To this end we examine a system where (H1) does not hold, i.e., where the control parameter varies the dynamics within the invariant subspace as well as that normal to it. We see that this has the effect of "spreading" the blowout bifurcation out over an interval of parameter values due to the existence of periodic windows where (H2) does not hold; however, we conjecture that there is a positive measure subset of parameters on which the blowout resembles the case for normal parameters.

The model we describe in Sec. II arises as the truncation of a stellar axisymmetric mean field dynamo model where the natural control parameters are not normal. There is also more than one invariant manifold forced by the spatial symmetries of the system, although this does not appear to affect the behavior in the cases examined, in the sense that it is only one of these manifolds, namely the antisymmetric one, which seems to dominate the attracting dynamics.

In Sec. III we discuss numerical results from simulations of the model; we discuss the basic bifurcational behavior in the full system and the antisymmetric subspace before discussing examples of type I intermittency, transient on-off intermittency and non-normal parameter blowout bifurcation in the model. In Sec. IV the implications of the results are discussed for more general systems of this type as well as for the dynamo problem. 


\section{MODEL}

\section{A. Galerkin model for the mean-field dynamo}

The dynamo model considered here is the so-called $\alpha \Omega$ mean-field dynamo model, with a dynamic $\alpha$ effect, given by Schmalz and Stix [8] (see also Covas et al. [9] for details). We assume a spherical axisymmetrical configuration with one spatial dimension $x$ (corresponding to the latitude and measured in terms of the stellar radius $R$ ) for which the magnetic field takes the form

$$
\vec{B}=\left(0, B_{\phi}, \frac{1}{R} \frac{\partial A_{\phi}}{\partial x}\right),
$$

where $A$ is the $\phi$ component (latitudinal) of the magnetic vector potential and $B$ is the $\phi$ component of $\vec{B}$. The model is given by the mean-field induction equation

$$
\frac{\partial \vec{B}}{\partial t}=\nabla \times\left(\vec{v} \times \vec{B}+\alpha \vec{B}-\eta_{t} \nabla \times \vec{B}\right),
$$

where $\vec{B}$ is the mean magnetic field, $\vec{v}$ is the mean velocity, $\eta_{t}$ is the turbulent magnetic diffusitivity, and $\alpha$ is the coefficient of the $\alpha$ effect [10]. In addition, the $\alpha$ effect, which is important in maintaining the dynamo action by relating the mean electrical current arising in helical turbulence to the mean magnetic field, is assumed to be dynamic and expressible in the form $\alpha=\alpha_{0} \cos x-\alpha_{M}(t)$, where $\alpha_{0}$ is a constant and $\alpha_{M}$ is its dynamic part satisfying the equation

$$
\frac{\partial \alpha_{M}}{\partial t}=\nu_{t} \frac{\partial^{2} \alpha_{M}}{\partial x^{2}}+Q \vec{J} \cdot \vec{B}
$$

where $Q$ is a physical constant, $\vec{J}$ is the electrical current, and $\nu_{t}$ is the turbulent diffusivity.

These assumptions allow Eq. (1) to be split into the following two equations:

$$
\begin{gathered}
\frac{\partial A_{\phi}}{\partial t}=\frac{\eta_{t}}{R^{2}} \frac{\partial^{2} A_{\phi}}{\partial x^{2}}+\alpha B_{\phi}, \\
\frac{\partial B_{\phi}}{\partial t}=\frac{\eta_{t}}{R^{2}} \frac{\partial^{2} B_{\phi}}{\partial x^{2}}+\frac{\omega_{0}}{R} \frac{\partial A_{\phi}}{\partial x} .
\end{gathered}
$$

Expressing these equations in a nondimensional form, relabeling the new variables to

$$
\left(A_{\phi}, B_{\phi}, \alpha_{M}\right) \Rightarrow(A, B, C),
$$

and using a spectral expansion of the form

$$
\begin{aligned}
& A=\sum_{n=1}^{N} A_{n}(t) \sin n x, \\
& B=\sum_{n=1}^{N} B_{n}(t) \sin n x, \\
& C=\sum_{n=1}^{N} C_{n}(t) \sin n x,
\end{aligned}
$$

where $N$ determines the truncation order, reduces Eqs. (2)(4) to a set of ordinary differential equations, the dimension of which depends on the truncation order $N$. We consider the full system given in terms of the variables $A_{n}, B_{n}, C_{n}$, $n=1, \ldots, N$ in the form

$$
\begin{gathered}
\frac{\partial A_{n}}{\partial t}=-n^{2} A_{n}+\frac{D}{2}\left(B_{n-1}+B_{n+1}\right) \\
+\sum_{m=1}^{N} \sum_{l=1}^{N} F(n, m, l) B_{m} C_{l}, \\
\frac{\partial B_{n}}{\partial t}=-n^{2} B_{n}+\sum_{m=1}^{N} G(n, m) A_{m}, \\
\frac{\partial C_{n}}{\partial t}=-\nu n^{2} C_{n}-\sum_{m=1}^{N} \sum_{l=1}^{N} H(n, m, l) A_{m} B_{l},
\end{gathered}
$$

where

$$
F(n, m, l)=\frac{8 n m l}{\pi(n+m+l)(n+m-l)(n-m+l)(n-m-l)},
$$

if $n+m+l$ is odd and $F(n, m, l)=0$ otherwise,

$H(n, m, l)=\frac{4}{\pi} \frac{n m l\left(-n^{2}+3 m^{2}+l^{2}\right)}{(n+l+m)(n+l-m)(n-l+m)(n-l-m)}$,

if $n+m+l$ is odd and $H(n, m, l)=0$ otherwise and

$$
G(n, m)=\frac{4 n m}{\pi\left(n^{2}-m^{2}\right)},
$$

if $n+m$ is odd and $G(n, m)=0$ otherwise. In these equations the control parameters are the so-called dynamo number $D$ (which is proportional to the square of the angular velocity gradient and to the square of the turnover time of the turbulent convection eddies) and the diffusivity ratio $\nu=\nu_{t} / \eta_{t}$.

\section{B. Invariant subspaces for the model with $N=4$}

Covas et al. [9] confined themselves to the study of models that are antisymmetric with respect to the equator and found that the minimum truncation order $N$ for which a similar asymptotic behavior existed was $N=4$. In this case, the equations have a twelve-dimensional phase space and are symmetric under the four-element Abelian group that comprises the identity $I$, the reversal transformation

$$
A_{n}(t) \rightarrow-A_{n}(t), \quad B_{n}(t) \rightarrow-B_{n}(t), \quad C_{n}(t) \rightarrow C_{n}(t),
$$

the antisymmetric (or dipolar) transformations

$$
\begin{gathered}
A_{n}(t) \rightarrow(-1)^{n+1} A_{n}(t), \quad B_{n}(t) \rightarrow(-1)^{n} B_{n}(t), \\
C_{n}(t) \rightarrow(-1)^{n} C_{n}(t)
\end{gathered}
$$

and the symmetric (or quadrupolar) transformations

$$
\begin{gathered}
A_{n}(t) \rightarrow(-1)^{n} A_{n}(t), \quad B_{n}(t) \rightarrow(-1)^{n+1} B_{n}(t), \\
C_{n}(t) \rightarrow(-1)^{n} C_{n}(t) .
\end{gathered}
$$


The trivial solution, given by $A_{n}=B_{n}=C_{n}=0$, is the only one that possesses both the dipolar and quadrupolar symmetries while symmetric solutions come in pairs and asymmetric solutions come in quadruples.

The antisymmetric part of these equations, which is obtained by putting $B_{1}=C_{1}=A_{2}=B_{3}=C_{3}=A_{4}=0$, was studied in [11]. We refer to this dynamically invariant subspace

$$
M_{A}=\left\{\left(A_{1}, 0,0,0, B_{2}, C_{2}, A_{3}, 0,0,0, B_{4}, C_{4}\right)\right\}
$$

as the antisymmetric subspace. There is also a sixdimensional symmetric invariant subspace

$$
M_{S}=\left\{\left(0, B_{1}, 0, A_{2}, 0, C_{2}, 0, B_{3}, 0, A_{4}, 0, C_{4}\right)\right\}
$$

although as we will see, the attractors are typically not contained within $M_{S}$. Throughout the paper we refer to the full system as the twelve-dimensional system.

\section{DYNAMICAL BEHAVIOR}

The system considered here has a two-dimensional parameter space $(D, \nu)$, neither of which is normal for the system restricted to $M_{A}$, as can be seen from Eqs. (5)-(7). We confine $\nu$ to the range $[0,1]$ on physical grounds, as otherwise there will be no dynamo action. Previous studies of these models have taken $\nu=0.5$. Here we shall consider two distinct cases of $\nu$ given by 0.5 and 0.47 in this range and in each case allow $D$ to vary. To study the dynamics of this system, we start by looking at the dynamics on the antisymmetric invariant submanifold $M_{A}$ and then look at how this changes as the full system is switched on.

\section{A. Basic bifurcation behavior}

To begin with, we consider the case of $\nu=0.5$ and as a first step make a coarse study of the dynamics confined to $M_{A}$ as well as the full (12-dimensional) system by considering the averaged energy $\left(E \propto \int_{0}^{\pi}|\vec{B}|^{2} d x\right)$ as a function of the parameter $D$. The results of these calculations are summarized in Figs. 1 and 2, respectively. The figures were produced using a fourth order variable step size Runge-Kutta method to integrate a number of randomly selected initial conditions forward in time, and so get a selection of the possible attractors. After a time when transients were deemed to have decayed (which we took to be 1000 time units) we averaged the energy over a much longer time series i.e., 10000 time units. We have verified the following results using the continuation package AUTO97 [12].

For small $D(D<98.67)$ all attracting dynamics of the full twelve-dimensional system is confined to the sixdimensional antisymmetric invariant submanifold $M_{A}$. The details of bifurcations are depicted in Figs. 1 and 2. As can be seen from these figures, as $D$ is increased, the fixed point at the origin (the trivial solution for both systems) bifurcates at $D=12.57$ to two fixed points, which are symmetric with respect to $A_{n} \rightarrow-A_{n}, B_{n} \rightarrow-B_{n}, C_{n} \rightarrow C_{n}$. A subcritical pitchfork-type bifurcation stabilizes the origin from $D=77.25$ up to a supercritical Hopf bifurcation at $D=98.67$.

For larger values of $D$ the two systems will evolve differently. In $M_{A}$ the supercritical Hopf bifurcation creates a stable periodic solution while the twelve-dimensional system

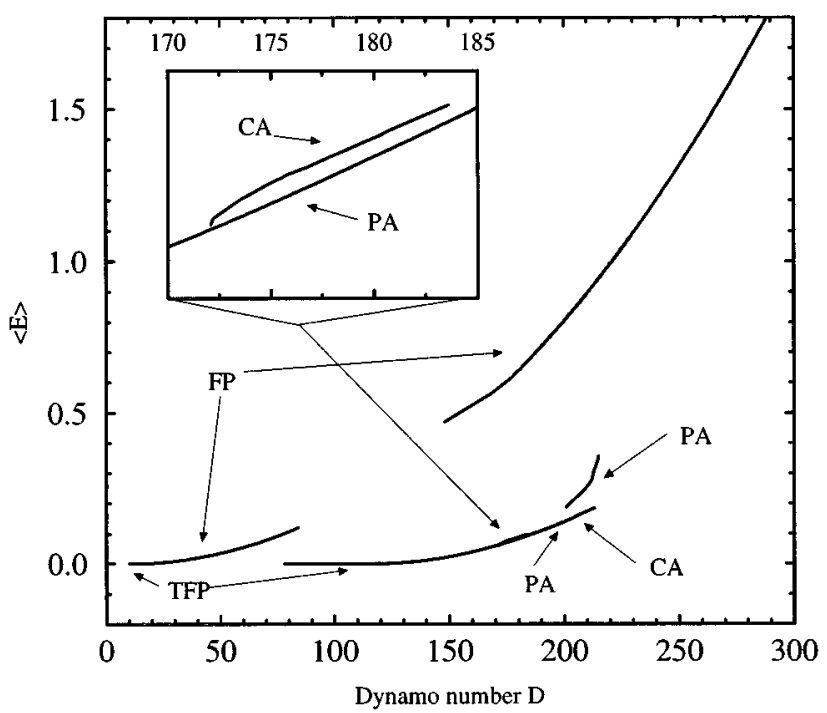

FIG. 1. Diagram showing attractors for a random selection of initial conditions within $M_{A}$ for $\nu=0.5$. TFP stands for trivial fixed point, FP for nontrivial fixed point, PA for antisymmetric periodic orbit, and CA for antisymmetric chaotic orbit. Continuation using DSTOOL shows that the break in the FP attractor uppermost in this diagram is just a feature of the choice of initial conditions; in fact it continues to be attracting over the whole range of $D$. The inset shows coexisting chaotic and periodic attractors over a range of $D$.

evolves on a branch of stable periodic solution with quadrupolar symmetry that becomes unstable through a torus bifurcation at $D=118.76$. In addition to this, at $D=118.23$, the origin undergoes Hopf bifurcation creating a branch of dipolar periodic solutions that are stabilized by a torus bifurcation at $D=127.78$, as shown in the inset of Fig. 2. Also shown in the inset is the appearance through a saddle-node bifurcation

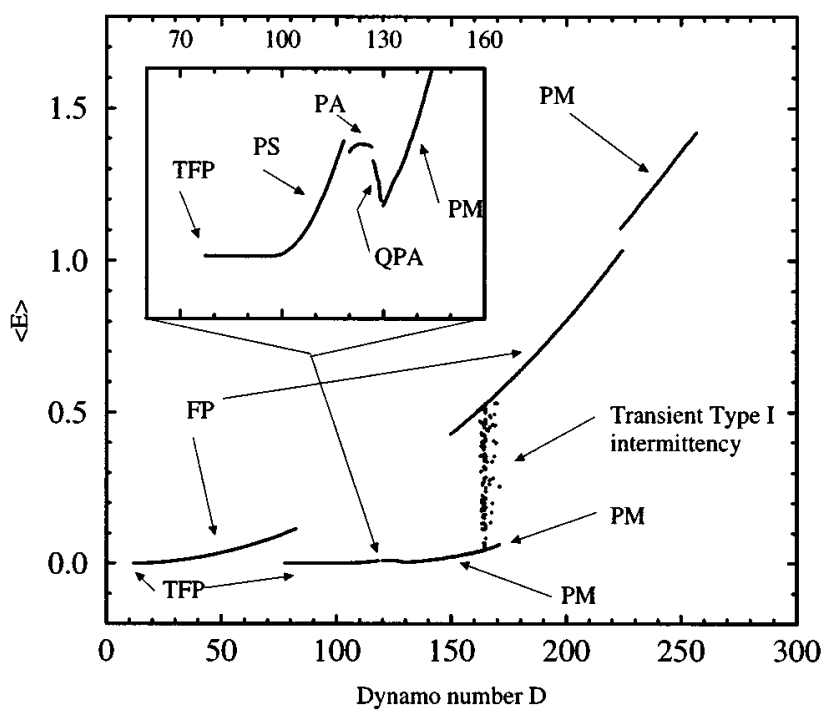

FIG. 2. Diagram showing attractors for randomly chosen initial conditions in the full phase space for $\nu=0.5$. PS stands for symmetric periodic orbit (i.e., in $M_{S}$ ), PM for a periodic orbit neither in $M_{A}$ nor in $M_{S}$, and QPA for antisymmetric quasiperiodic orbit. The notations of TFP, FP, and PA are as in Fig. 1. Observe the existence of intermittent behavior over a range of $D$. 


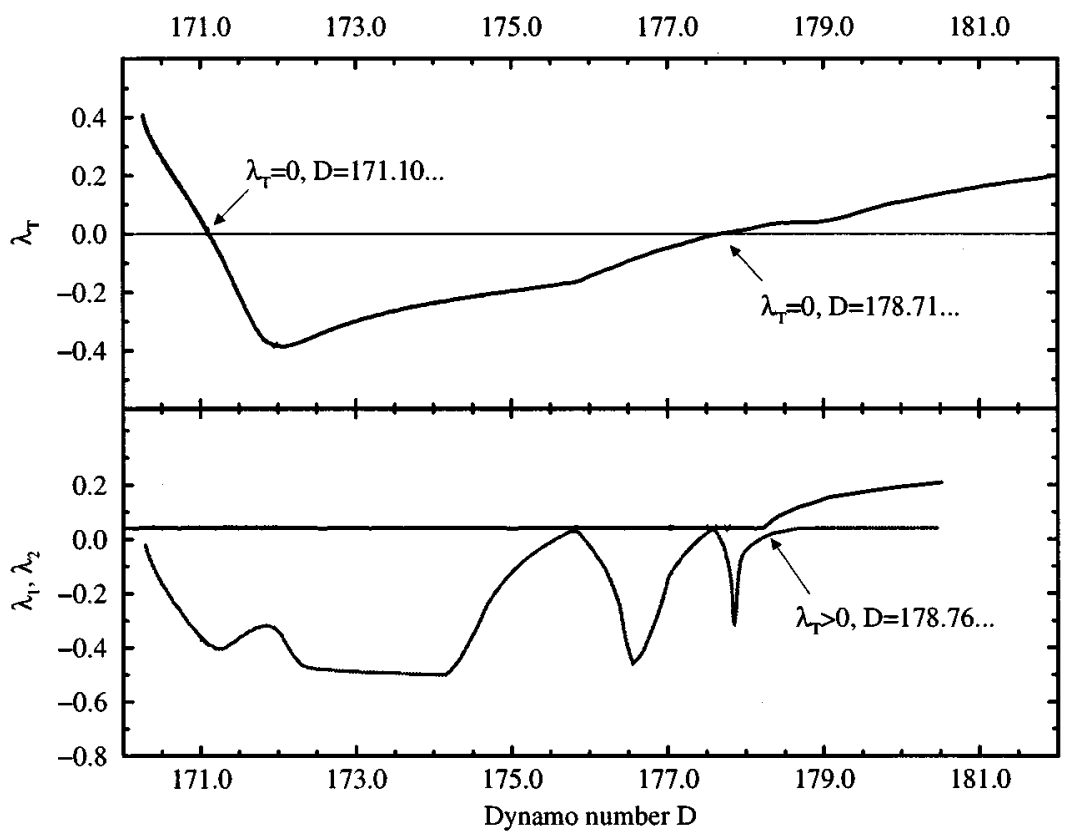

FIG. 3. The leading (i.e., most positive) transverse Lyapunov exponent and the two leading Lyapunov exponents for the attractor of a particular initial condition for the system on $M_{A}$ at $\nu=0.5$. The attractor is a periodic orbit from $D=170.25$ up to $D=178.76$, although it undergoes a number of period doublings in this range to give a chaotic attractor for $D>178.76$. The computed orbit is transversely stable in the range $171.10<D<178.71$. By reducing $\nu$ to 0.47 we can change order of the loss of transverse stability and the breakdown to chaos in $M_{A}$.

of a branch of periodic solutions in $M_{S}$ at $D=132.72$ that are stable up to $D=135.42$. Here they lose stability at a torus bifurcation. All of these branches of solutions pass through the region from $D=150$ to $D=175$ without bifurcation; however, they do not provide a complete picture of all that happens in this region.

There is a pair of periodic orbits in $M_{A}$ that are created at a saddle-node bifurcation at $D=170.25$. These are stabilized by a pitchfork bifurcation of periodic orbits at $D=171.10$ creating a branch of stable periodic orbits with no symmetry continuing down to $D=171.003$; these are important for the intermittent dynamics discussed in the next subsection (Fig. 4). For larger $D$, the periodic orbit undergoes a sequence of bifurcations preserving the dipolar symmetry (i.e., creating branches that remain within $M_{A}$ ) up until $D=177.75$ where a branch of stable periodic orbits that bifurcate from $M_{A}$ is created. We conjecture that the saddle node bifurcation creating this series of periodic orbits is associated with breakdown of a quasiperiodic flow on a two-torus created at one of the torus bifurcations but have not been able to check this.

\section{B. Intermittent dynamics}

We investigated two cases, $\nu=0.5$ and $\nu=0.47$. Note that for physically meaningful results we require $\nu \leqslant 0.5$. We have examined the transverse stability of attractors in $M_{A}$ by calculating the corresponding transverse Lyapunov exponent $\left(\lambda_{T}\right)$. Figure 3 summarizes the results of calculations of the $\lambda_{T}$ for the periodic orbit which is created at $D=170.25$, as shown in the inset in Fig. 1. The important feature in this behavior of $\lambda_{T}$ is the presence of the two crossings through zero. The transverse stability of the other attractors does not change in this range of $D$.

\section{The case of $\nu=0.5$}

For the case $\nu=0.5$ we examined the behavior of the full system over a parameter interval in the neighborhood of $D=171$. The loss of stability of the periodic orbit described in the previous section does not induce on-off intermittency as we first suspected. Instead, the bifurcation at $D=171.10$ is a pitchfork bifurcation that creates an asymmetric periodic orbit that survives up to $D=171.003$ and is then destroyed by a collision with an unstable orbit in a saddle node bifurcation, which is shown in Fig. 4.

For $D<171.003$, we have a transient type-I intermittency, as can be seen in Fig. 5. We also calculated the scalings of the transient times and average times between the bursts (as shown in Fig. 6 and Fig. 7) and found them to be in good agreement with the known $-1 / 2$ scaling. The behavior between bursts shown in Fig. 5, resembles the 12D periodic orbit discussed above, except that the amplitude of the symmetric part of the oscillations between the bursts grows slowly towards the bursts and returns, after the bursts, close to the invariant submanifold. The intermittent behavior is transient, in the sense that the orbit returns to the fixed point in the invariant submanifold. The spectrum of Lyapunov exponents for these transient forms of intermittency is in the form $(+, 0,-, \ldots)$, until the transient dies out, becoming attracted to a stable fixed point (note that there are two such fixed points, located symmetrically with respect to the $A$ and $B$ variables).

On the negative side of the crossing of the transverse Lyapunov exponent, shown in Fig. 3, we observe a basin boundary for the full twelve-dimensional system with a dimension close to that of the phase space. This is shown in Fig. 8, which demonstrates which asymptotic attractor on the 


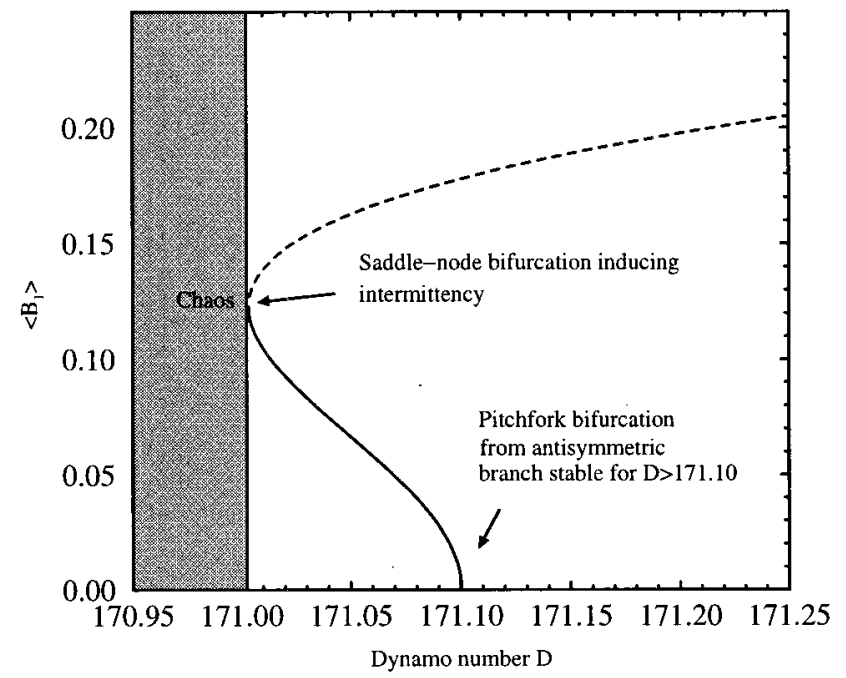

FIG. 4. Continuation of a periodic orbit showing breakdown to type I intermittency at $\nu=0.5$. The abscissa shows a symmetric component of a branch of PM periodic orbits created at a pitchfork of a PA periodic orbit. This is destroyed at a saddle-node bifurcation giving rise to type I intermittency at $D<170.003$.

invariant submanifold the initial conditions get attracted to. Both basins seem to be made up of open sets (supported by the fact that calculations indicate an integer box counting dimension). This conclusion is further supported by the calculation of the exterior dimension [13] shown in Fig. 9.

It is also interesting to note, from both physical and mathematical points of view, that even if the full (twelvedimensional) system does have new attractors, nevertheless it will in general alter the relative size of the basins of attraction; most initial points seem to get attracted to only the fixed

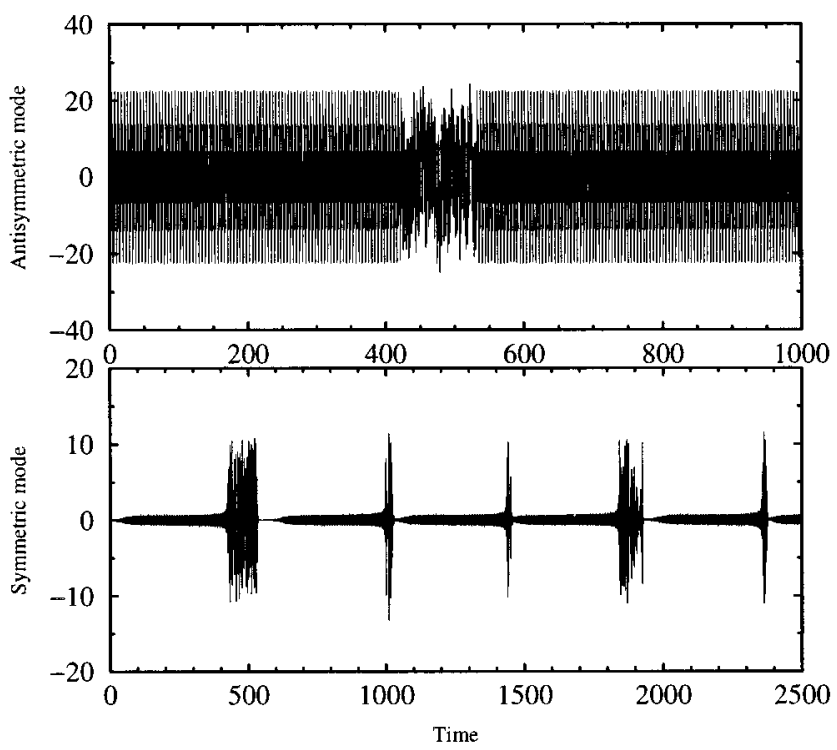

FIG. 5. Time series showing a component transverse to $M_{A}$ for transient type I intermittency series for $\nu=0.5$ at $D=170$. Observe the long but irregular periods of lingering near a small amplitude periodic orbit interspersed by large fluctuations. After a long time, the trajectory is asymptotic to a stable fixed point.

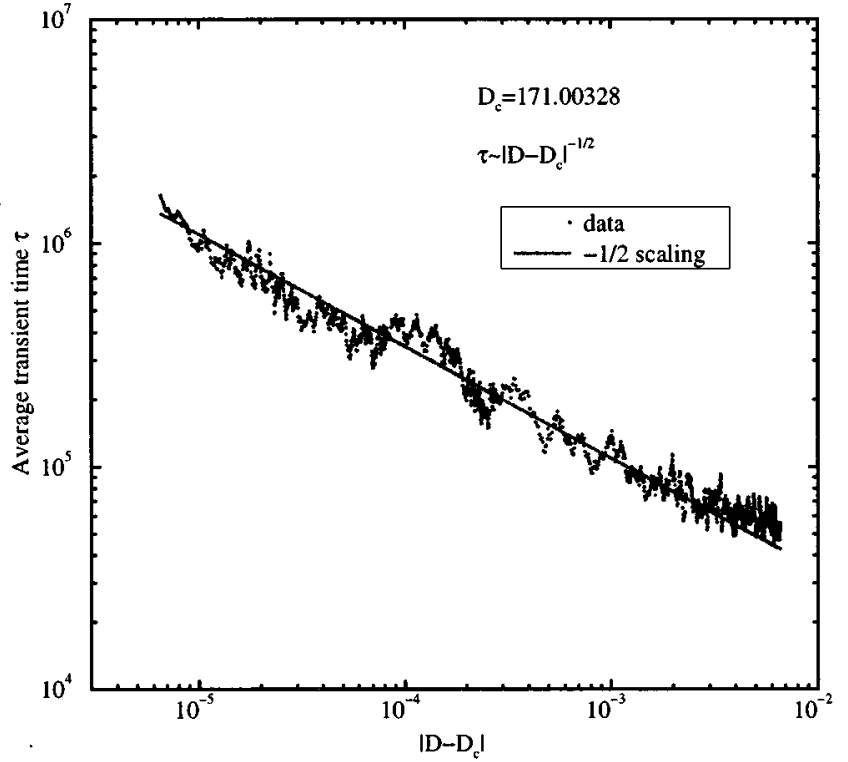

FIG. 6. Scaling of transient time of the transient type I intermittency for $\nu=0.5$ against the unfolding parameter $D-D_{c}$.

points or one of the cycles, as opposed to initial conditions starting in the invariant submanifold.

\section{The case of $\nu=0.47$}

By making $\nu$ slightly smaller than 0.5 we were able to change the order of the bifurcation to chaos in the invariant submanifold relative to the loss of transverse stability. To study the behavior of the system with $\nu=0.47$, we looked at the parameter region in which the system with $\nu=0.5$ had a chaotic attractor (as depicted in Fig. 3). For our calculations, we chose our initial conditions to lie in the basin of the chaotic attractors for $\nu=0.5$ system. We then studied the evolution of the system for $\nu=0.47$ by changing the control parameter and taking the initial conditions at each step to lie

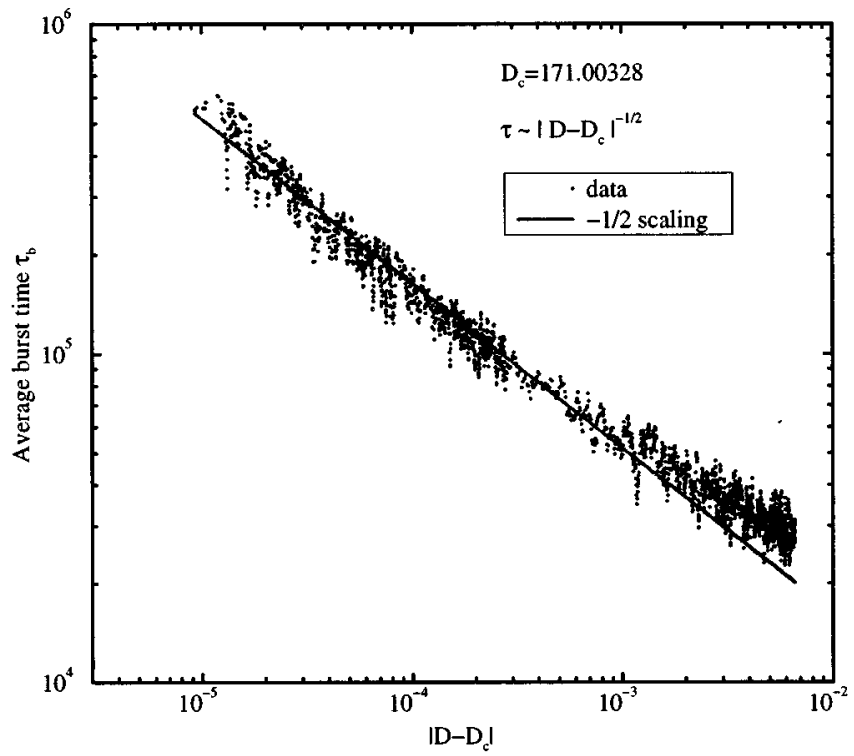

FIG. 7. Scaling of transient burst time of the transient type I intermittency for $\nu=0.5$. 


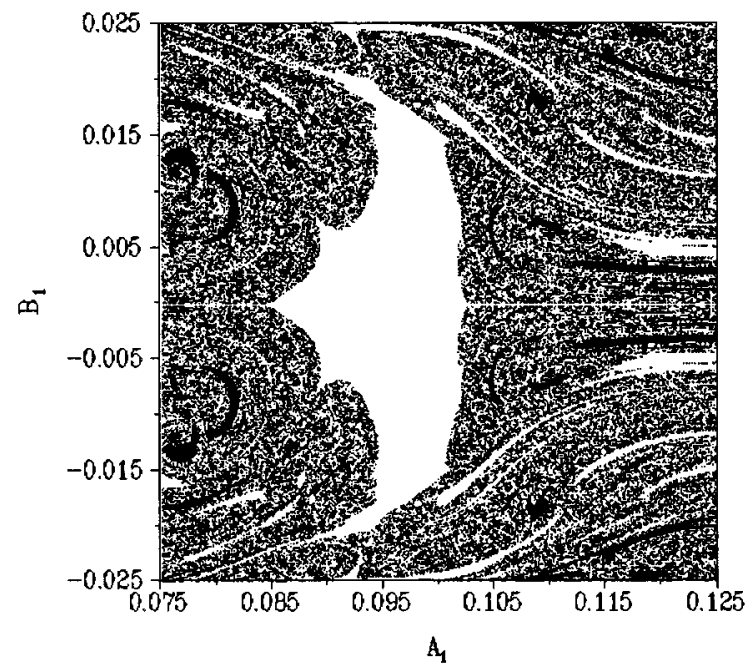

FIG. 8. Two-dimensional slice through phase space obtained by setting all components to zero except for $A_{1}$ and $B_{1}$. The basins of attraction of the fixed point (black) and the periodic orbit (white) that are coexisting attractors at $D=171.12$ and $\nu=0.5$.

in the basin of the attractor for the previous parameter value. The results of these calculations for the $\lambda_{T}$ and the largest Lyapunov exponents of the full twelve-dimensional system are given in Figs. 10 and Fig. 11. As can be seen, the chaotic behavior is now interspersed with periodic windows. Within these windows the periodic solutions coexist with chaotic repellers.

Another crossing of the transverse Lyapunov exponent, from negative to positive, shown in Fig. 3, occurs at $D=178.71$, and for $177.10<D<178.71$ there are stable periodic orbits restricted to the invariant submanifold. This crossing is also related to a bifurcation of periodic orbits and therefore there is no indication of on-off intermittency or

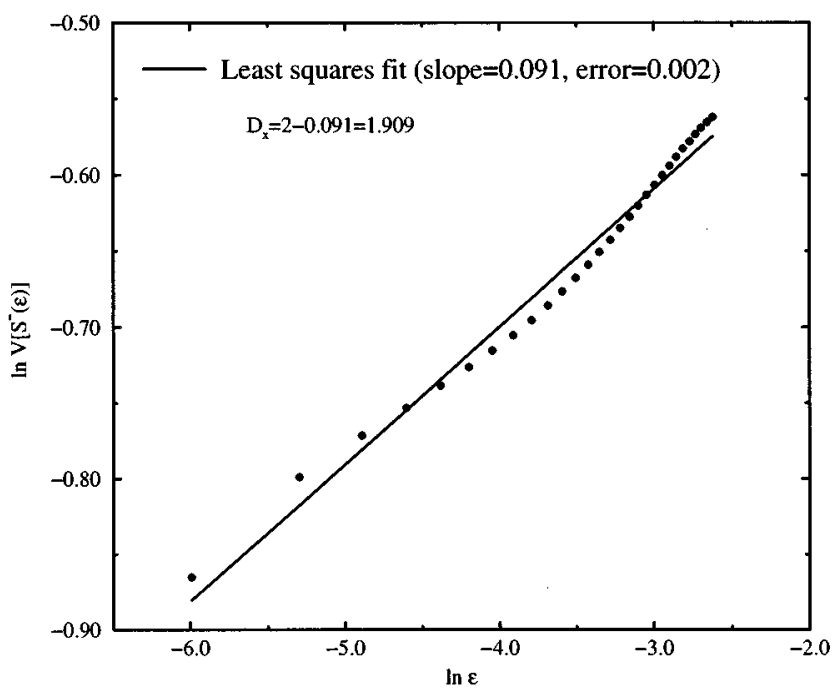

FIG. 9. Approximation of the exterior dimension $D_{x}$ of the basin shown in black in Fig. 8. This is very close to the dimension of the slice through phase space indicating that the basin boundary is highly convoluted, even though it is not riddled.

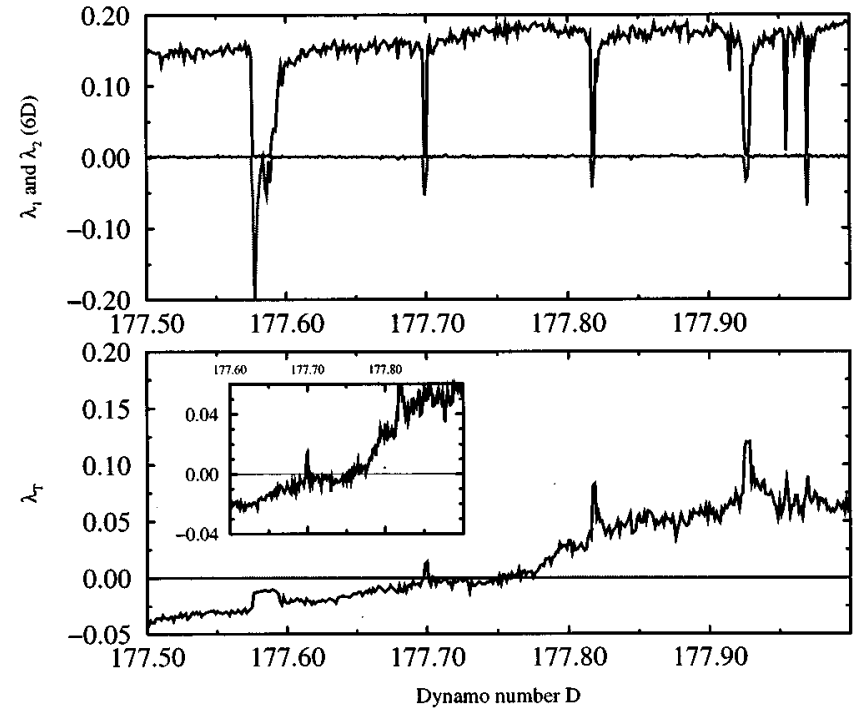

FIG. 10. Largest transverse Lyapunov exponent $\left(\lambda_{T}\right)$ and the two leading Lyapunov exponents $\left(\lambda_{1}\right.$ and $\left.\lambda_{2}\right)$ for a family of attractors on the antisymmetric invariant submanifold for $\nu=0.47$. Note that the existence of periodic windows and the general trend of $\lambda_{T}$ through zero indicate a blowout bifurcation near $D \approx 177.75$. The lack of smoothness of these curves is indicative of the fact that $D$ is not a normal parameter.

riddled basins. The periodic orbit on the invariant submanifold becomes chaotic just after $D=178.76$, not close enough to the transverse stability bifurcation at $D=177.71$ to induce on-off intermittency. This suggests that there is likely to be a blowout at nearby parameters in the two parameter space, as we discuss in the next section. To substantiate this, we calculated the scaling of the probability distribution of the off phases (corresponding to when the distance to the invariant submanifold is less than $10^{-3}$ ) as a function of their length.

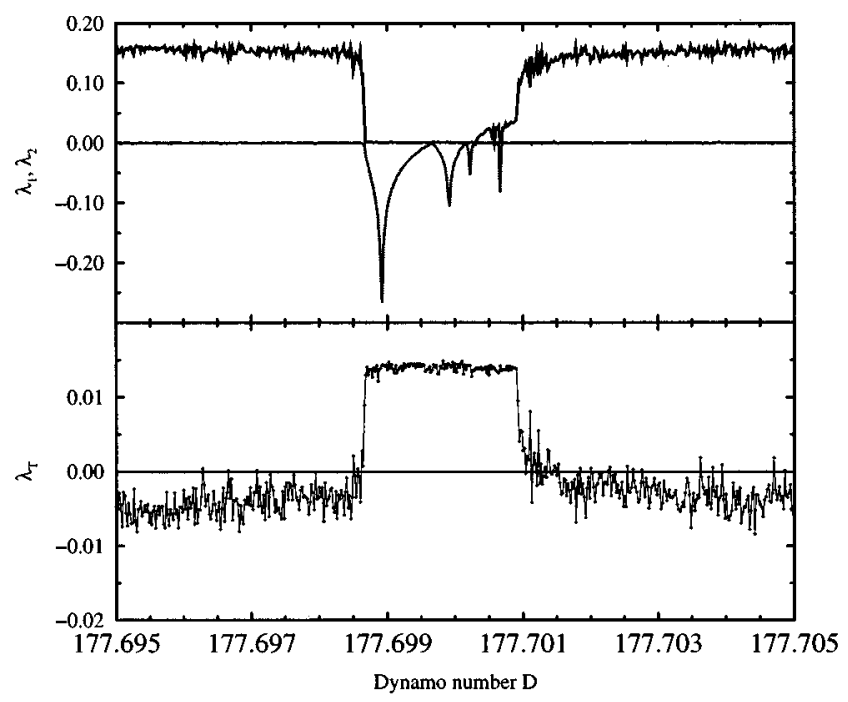

FIG. 11. Amplification of the transverse Lyapunov exponent and the two leading Lyapunov exponents for the antisymmetric subset of equations for $\nu=0.47$. This shows the existence of a "window" in parameter space where the attractor within the invariant subspace is periodic and transversely repelling. 


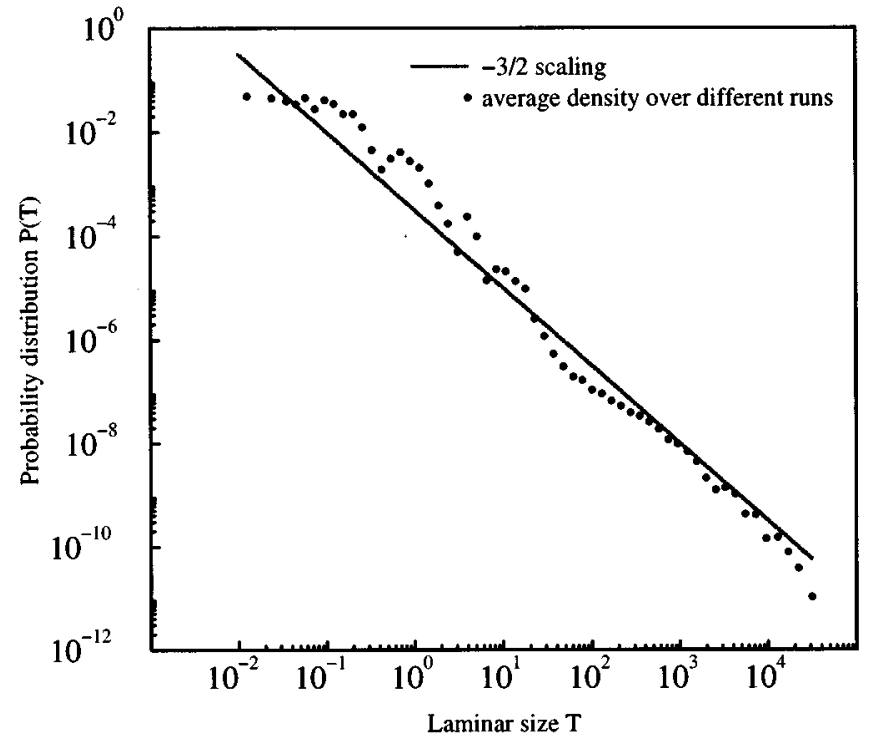

FIG. 12. Scaling of the laminar phases over an on-off transient orbit segment for $\nu=0.47$ and $D=177.70$. The $-3 / 2$ scaling is evidence of an on-off intermittent state.

As can be seen in Fig. 12, the scaling agrees with the powerlaw behavior proposed in [2] with an index of $-3 / 2$. At this parameter value transient on-off trajectories appear to be induced by a chaotic invariant set that is a repeller within the invariant submanifold.

\section{Generic behavior for non-normal parameters}

There are a number of interesting dynamical phenomena that occur here that are related to the fact that the system parameters are not normal.

(I) The chaotic behavior in the invariant submanifold appears to be of the nonuniformly hyperbolic variety, and in particular the chaotic attractors are not structurally stable; they are destroyed by arbitrarily small perturbations. Notwithstanding this, we find numerical evidence (Figs. 10 and 11) that there is a family of chaotic attractors with similar properties defined on a subset of parameter space with positive (Lebesgue) measure but open (even dense) complement. This is what is found, for example, in the logistic map [14]. In the open dense complement we expect to see periodic windows and many bifurcations, for example, period doubling cascades, which we have found numerically. This is consistent with the conjecture of Barreto et al. [16] on noting that the attractors here have only one positive Lyapunov exponent. In this parameter region the system may be said to be fragile [17], in the sense that arbitrarily small changes in the control parameter $D$ can force a chaotic attractor to be replaced by a nearly attracting periodic orbit.

(II) In the light of (I), there is no reason why there should be a unique parameter value $D_{c}$ at which blowout occurs. In particular, the attractor in $M_{A}$ varies discontinuously, and its tangential and normal Lyapunov exponents vary discontinuously with $D$ except within the periodic windows. This explains the presence of smooth segments in the curves of Fig. 11 within regions where the attracting dynamics is periodic.

(III) In this system the passage of $\lambda_{T}$ through zero is fairly

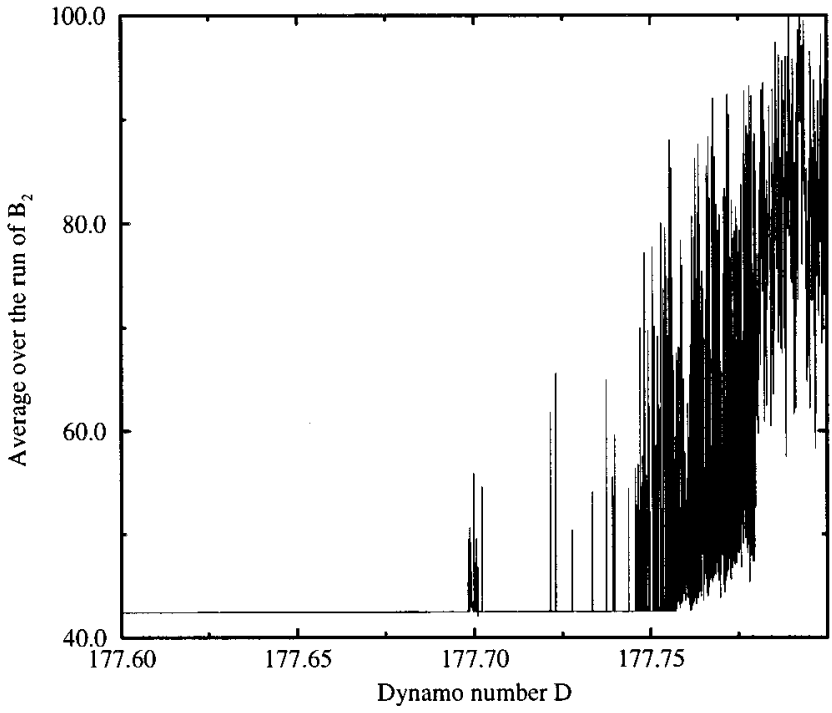

FIG. 13. Average of the variable $B_{2}$ measuring average distance from $M_{A}$ over an on-off transient orbit segment that eventually ends at a fixed point (for $\nu=0.47$ ). The discontinuous nature of this presumably reflects the discontinuous change in $\lambda_{T}$ illustrated in Fig. 10.

simple. Firstly, the value of $\lambda_{T}$ can be bracketed between upper and lower bounds that also pass through zero. This is presumably due to the fact that certain periodic orbits in the attractor will typically maximize and minimize transverse Lyapunov exponents [15]. Secondly, there is evidence that there is a positive measure Cantor set $\mathcal{S}$ in parameter space where chaotic behavior exists. On $\mathcal{S}$ the Lyapunov exponents are continuous in the sense that there is a continuous function $\lambda^{\prime}$ of parameter that is equal to $\lambda_{T}$ on $\mathcal{S}$ and passes through zero at about $D=177.75$ (see Fig. 10 and also Fig.

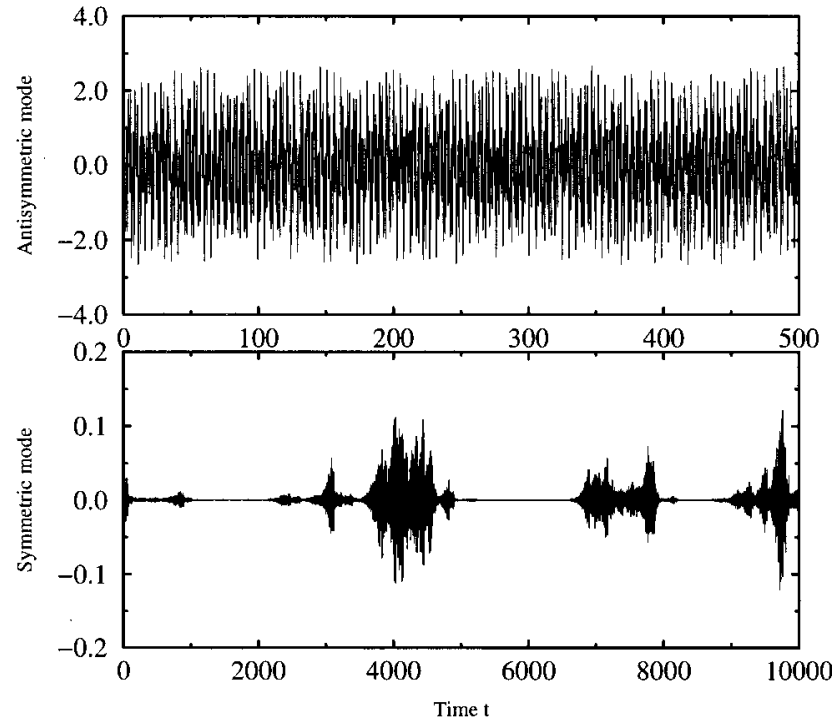

FIG. 14. Time series showing transient on-off intermittency for $\nu=0.47$ and $D=177.70$. The on-off intermittent behavior is induced by a chaotic repeller that is present within the periodic windows. After a long transient, the trajectory is asymptotic to a stable fixed point within $M_{A}$ (not shown). 
13). Even for parameter values not in $\mathcal{S}$ we can get transient on-off intermittent behavior (see Fig. 14).

On the basis of our results we conjecture that properties (I) and (II) are typical behavior at blowout on varying a non-normal parameter and (III) is a typical simple scenario of how this can occur.

For the case $\nu=0.47$ we note that the transverse Lyapunov exponent (of the chaotic invariant set that attracts within $M_{A}$ ) becomes positive and causes the appearance of transient on-off intermittency.

\section{DISCUSSION}

We have studied global dynamics and bifurcations occurring in a twelve-dimensional truncation of a stellar meanfield dynamo model that possesses two six-dimensional invariant submanifolds corresponding to dipolar and quadrupolar symmetries, respectively. An essential feature of this model is that its control parameters are non-normal, allowing the dynamics to vary both within the invariant submanifolds as well as in the directions normal to them.

Depending upon the region of the parameter space considered, we find a diverse set of dynamical modes of behavior, including different forms of intermittency. In addition to transient type I intermittency, we find transient on-off intermittency induced by blowout bifurcations. In the parameter range where we observe the latter behavior, the invariant submanifold possesses a family of chaotic attractors on a subset of parameter space with positive (Lebesgue) measure but open (even dense) complement. On the basis of our numerical calculations these attractors seem to be structurally unstable, which is consistent with the conjecture of Barreto et al. [16]. We also find that as a consequence of the nonnormality of the control parameters the blowout bifurcation seems to occur over an interval rather than a point in the parameter space.

These results can be of potential significance for the dynamical behavior of systems with non-normal parameters. Given the fact that the model considered here was derived directly from dynamo equations, the forms of intermittency found here can also be of potential importance in understanding the mechanism of production of the so-called grand or Maunder-type minima in solar and stellar activity, during which the amplitudes of stellar cycles are greatly diminished $[18,19]$. We do not, however, wish to imply that the forms of intermittency responsible for such stellar behavior are necessarily transient.

\section{ACKNOWLEDGMENTS}

E.C. was supported by Grant BD/5708/95-Program PRAXIS XXI, from JNICT-Portugal. P.A. was partially supported by a Nuffield "Newly appointed science lecturer" grant. R.T. benefited from PPARC UK Grant No. H09454. This research also benefited from the EC Human Capital and Mobility (Networks) grant "Late type stars: activity, magnetism, turbulence" No. ERBCHRXCT940483.
[1] J. Alexander, I. Kan, J. Yorke, and Z. You, Int. J. Bifurcations Chaos 2, 795 (1992).

[2] M. Platt, E. Spiegel, and C. Tresser, Phys. Rev. Lett. 70, 279 (1993).

[3] E. Ott and J. Sommerer, Phys. Lett. A 188, 39 (1994).

[4] F. Xie and G. Hu, Phys. Rev. E 53, 1232 (1996).

[5] J. Milnor, Commun. Math. Phys. 99, 177 (1985).

[6] P. Ashwin, J. Buescu, and I. Stewart, Phys. Lett. A 193, 126 (1994); P. Ashwin, J. Buescu, and I. Stewart, Nonlinearity 9 , 703 (1996).

[7] T. Yal cinkaya and Y.-C. Lai, Phys. Rev. Lett. 77, 5039 (1996).

[8] S. Schmalz and M. Stix, Astron. Astrophys. 245, 654 (1991).

[9] E. Covas, A. Tworkowski, A. Brandenburg, and R. Tavakol, Astron. Astrophys. 317, 610 (1996).

[10] F. Krause and K.-H. Rädler, Mean Field Magnetohydrodynamics and Dynamo Theory (Pergamon, Oxford, 1980).

[11] E. Covas and R. Tavakol, Phys. Rev. E 55, 6641 (1997).

[12] E. Doedel, A. Champneys, T. Fairgrieve, Y. Kuznetsov, B.
Sandstede, and X. Wang, AUTO97: Continuation and bifurcation software for ordinary differential equations (available via FTP from directory pub/doedel/auto at ftp.cs.concordia.ca) (1997).

[13] For a discussion of exterior dimension see Y.-C. Lai and C. Grebogi, Phys. Rev. E 53, 1371 (1996) Ref. [3].

[14] M. V. Jakobson, Commun. Math. Phys. 81, 39 (1981).

[15] B. Hunt and E. Ott, Phys. Rev. Lett. 76, 2254 (1996).

[16] E. Barreto, B. Hunt, C. Grebogi, and J. Yorke, Phys. Rev. Lett. 78, 4561 (1997).

[17] We note in passing that the term fragile was used in essentially the same sense also in R. K. Tavakol and G. F. R. Ellis, Phys. Lett. A 130, 217 (1988).

[18] N. O. Weiss, in Lectures on Solar and Planetary Dynamos, edited by M. R. E. Proctor and A. D. Gilbert (Cambridge University Press, Cambridge, 1994).

[19] E. Spiegel, N. Platt, and C. Tresser, Geophys. Astrophys. Fluid Dyn. 73, 146 (1993). 